\title{
Lithium and rotation on the subgiant branch. A theoretical analysis of observations
}

\author{
J. D. do Nascimento $\mathrm{Jr}^{1,4}$, C. Charbonnel ${ }^{1}$, A. Lèbre ${ }^{2}$, P. De Laverny ${ }^{3}$ \\ and J. R. de Medeiros ${ }^{4}$ \\ ${ }^{1}$ Laboratoire d'Astrophysique de Toulouse, 16 Avenue E. Belin, 31400 \\ Toulouse, $\mathrm{Fr}$ \\ ${ }^{2}$ GRAAL, Université Montpellier II, F-34095 Montpellier Cedex, Fr \\ ${ }^{3}$ Observatoire de la Côte d'Azur, BP 4229, 06304 Nice CEDEX 4, Fr \\ ${ }^{4}$ Departamento de Fisica, Universidade Federal do Rio Grande do \\ Norte, 59072-970 Natal, R.N., Br
}

\begin{abstract}
Lithium abundances, determined for 120 subgiant stars in Lèbre et al. (1999), are analyzed and compared with predictions for dilution. To this purpose, the evolutionary status of the sample as well as the individual masses have been determined. We look for the distributions of $\mathrm{A}(\mathrm{Li})$ and $V \sin i$ with mass when those stars evolve along the subgiant branch. Our results bring a new light on the Lithium and rotation discontinuities in evolved phases.
\end{abstract}

We investigate the physical processes that underline the lithium and rotational discontinuities along the subgiant branch based on new high resolution spectroscopic observations and precise rotational velocities. We have analyzed the $\mathrm{Li}$ and rotation observations for 120 Pop I F, G and K spectral types subgiant stars. We use rotational velocities given by De Medeiros \& Mayor (1999) as well as the values derived by Lèbre et al. (1999) for $\log g, A_{\mathrm{Li}}$ and $T_{\text {eff }}$ with their respective errors. We use the HIPPARCOS parallax measurements to locate precisely our objects in the HR diagram and determine the individual mass and evolutionary status. The tracks, mass and evolutionary stage determinations for the sample of stars are explained in do Nascimento et al. (2000a). The lithium discontinuity simply reflects the well-known dilution that occurs when the convective envelope starts to deepen after the turnoff and reaches the inner free-lithium layers. The dilution is a fast process, both in terms of age and effective temperature interval. We have compared the observed $\mathrm{Li}$ abundances with predictions of $\mathrm{Li}$ dilution caused by the deepening of the convective envelope on the subgiant branch. Stars with masses $<1.2 \mathrm{M}_{\odot}$ show a large range in abundance before the turnoff, indicating lithium depletion in the previous phases. Stars with masses between 1.2 and $1.5 \mathrm{M}_{\odot}$ show $A_{\mathrm{Li}}$ values in agreement with what is found in the open clusters. Many stars with masses higher than $1.5 \mathrm{M}_{\odot}$ (Fig. 1c,d) show lithium depletion up to two orders of magnitude before the start of the dilution at $\log \mathrm{T}_{\text {eff }} \simeq 3.75$. Our observational result is in agreement with that one found by Balachandran (1990) for a few slightly evolved field stars originating from the hot side of the dip and showing 


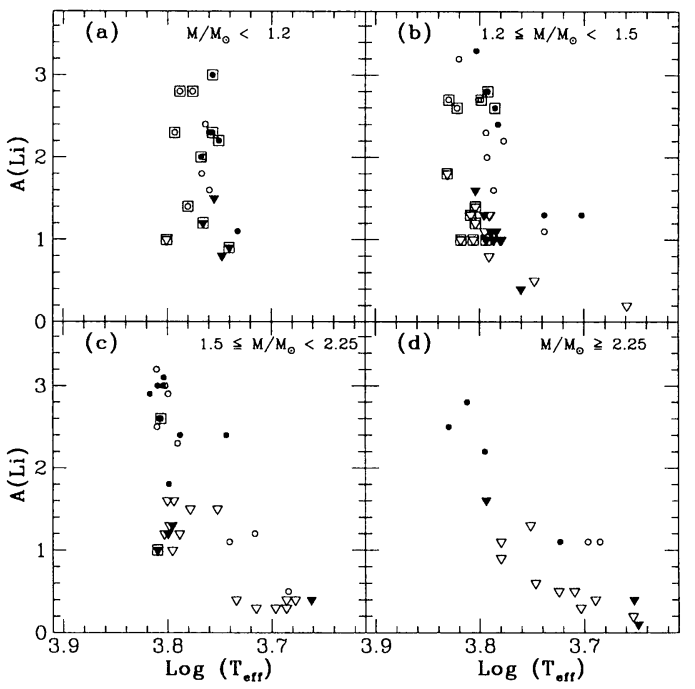

Figure 1. Lithium abundances as a function of $\log \left(\mathrm{T}_{\mathrm{eff}}\right)$ for all our sample stars. Open and filled symbols represent single and binary stars respectively. The circles correspond to lithium detection while inverted triangles are for upper limits in the lithium abundance determination. Squares point out the main sequence stars

significant lithium depletion. This confirm the suggestion by Vauclair (1991) that some extra-lithium depletion occurs inside these stars when they are on the main sequence, even if its signature does not appear in the stellar surface at the age of the Hyades. Among some effects available one can quote an influence of rotation-induced mixing limited by a self-regulated hydrodynamical process in the main-sequence (see do Nascimento et al. 2000b and Vauclair 2000, on this meeting). For the rotation, we confirm that low mass stars leave the main sequence with a low rotational rate, while more massive stars are slowed only when reaching the subgiant branch. Our interpretation shows that lithium and rotation discontinuities seems to be independent.

\section{References}

Balachandran, S. 1990 ApJ 354, 310

De Medeiros J.R., Mayor M. 1999 A\&AS 139, 443

do Nascimento, J.D.Jr, Charbonnel, C., Lèbre, A., de Laverny, P., de Medeiros, J.R., 1999, preprint (a)

do Nascimento, J.D.Jr., Vauclair, S. 2000, this conference (b)

Lèbre A., de Laverny P., De Medeiros J.R., Charbonnel C., da Silva L. 1999, A\&A, 345, 936

Vauclair, S. 1991, in IAU Symp. 145, Evolution of Stars: the Photospheric Abundance Connection (Michaud, G., Tutukov, A. eds.) p. 327

Vauclair, S. 2000, this conference 